Communications in Physics, Vol. 23, No. 2 (2013), pp. 127-133

\title{
UNCORRELATED ELECTRON-HOLE TRANSITION ENERGY IN GaN|InGaN|GaN SPHERICAL QDQW NANOPARTICLES
}

\author{
HADDOU EL GHAZI \\ LPS, Faculty of science, \\ Dhar EL Mehrez, BP 1796 Fes-Atlas, Morocco \\ and \\ Special mathematics, CPGE Kénitra, \\ Chakib Arsalane Street, Morocco \\ Email: hadghazi@gmail.com; Phone: (+212) 655194137 \\ ANOUAR JORIO AND IZEDDINE ZORKANI \\ LPS, Faculty of science, Dhar EL Mehrez, \\ BP 1796 Fes-Atlas, Morocco
}

Received 04 March 2013; revised manuscript received 03 April 2013 Accepted for publication 08 May 2013

\begin{abstract}
The electron (hole) energy and uncorrelated $1 S_{e}-1 S_{h}$ electron-hole transition in Core $(G a N)\left|\operatorname{well}\left(\operatorname{In}_{x} G a_{1-x} N\right)\right| \operatorname{shell}(G a N)$ spherical $Q D Q W$ nanoparticles are investigated as a function of the inner and the outer radii. The calculations are performed within the framework of the effective-mass approximation and the finite parabolic potential confinement barrier in which two confined parameters are taking account. The Indium composition effect is also investigated. A critical value of the outer and the inner ratio is obtained which constitutes the turning point of two indium composition behaviors.
\end{abstract}

\section{INTRODUCTION}

During the past few decades, quantum dot-quantum well (QDQW) nanoparticles have attracted much interest due to their importance in physics, electronic and optical applications [1-5]. The so-called QDQW nanoparticles are typically the spherical core-shell three-layer heterostructures composed of two different materials, in which the material with the smaller bulk band gap is embedded between a core and an outer shell made of the larger band gap material. This structure can be treated as a complicated QD semiconductor with a smaller band gap layer in its core acting as an internal well. This structure gives rise to a significant redistribution of the carriers. The nanoparticles QDQW provide a flexibility to tailor electron and hole characteristics by changing the core radius, the thickness of the well and the outer shell radius, which amounts to a three-dimensional example of properties engineering. Within the framework of the effective-mass approximation, Mews et al. [1] have investigated the $1 S_{e}-1 S_{h}$ electronic transition energy in $\mathrm{CdS}|\mathrm{HgS}| \mathrm{CdS}$ particles in which the coulomb interaction is ignored. Schooss et al. [2] investigated both theoretically and experimentally the coulomb interaction and the $1 S_{e}-1 S_{h}$ electronic transition energy considering the finite barrier at the nanoparticle boundary. 
Many others materials such ZnS|CdS|ZnS [6,7], ZnSe|InAs|ZnSe [8], GaAs|InAs|GaAs [9], $\mathrm{CdSe}|\mathrm{HgSe}| \mathrm{CdSe}[3]$ are reported. Recently, a typical structure based on symmetric QDQW CdSe $|\mathrm{ZnS}| \mathrm{CdSe}$ and asymmetric QD-QW ZnS|InP|ZnSe are studied respectively by Fang et al. [10] and Kazaryan et al. [11]. Additionally, Sadeghi and Rezaie [12] have studied the excited states electron-hole transition under the magnetic field in symmetric GaAs| $|(\mathrm{Al}, \mathrm{Ga}) \mathrm{As}| \mathrm{GaAs}$. Incidentally, we have investigated the electric field effect on the hydrogenic states in symmetric GaN|GaInN|GaN QD-QW [13].

It is well known that one of the most interesting novel features of QDQW nanoparticles is the electronic transition between electron and hole states localized in its internal well layer. This transition gives rise to a new band gap observed experimentally, referred to $1 S_{e}-1 S_{h}$ transition in $[1,2,14,15]$. So far, for a QDQW nanoparticles with a narrow well layer less than three monolayers in thickness, all of the reported experimental results have concentrated on the $1 S_{e}-1 S_{h}$ electronic transition [1,2]. Thus, in this paper we have concentrated on the $1 S$ state for electron and hole confined in a parabolic potential QDQW nanoparticle.

\section{THEORETICAL FRAMEWORK}

\section{II.1. Finite parabolic potential barrier}

Throughout this paper, the units of length is given in terms of effective Bohr radius

$a_{B}=\frac{\varepsilon_{0} \hbar^{2}}{m^{*} e^{2}}$. The schematic description and the corresponding potential energies of the studied structure are depicted in Fig. 1. From left to right one sees the GaN core for $0 \prec r \prec R_{1}$, the $\operatorname{In}_{x} \mathrm{Ga}_{1-x} \mathrm{~N}$ well for $R_{1} \leq r \leq R_{2}$ and the GaN shell for $R_{2} \prec r$. The hydrogenic shallow-donor impurity is located in the spherical quantum dot-quantum well center made out of $\mathrm{GaN}$ (core) $\mid \mathrm{In}_{x} \mathrm{Ga}_{1-x} \mathrm{~N}$ (well) $\mid \mathrm{GaN}$ (shell) .

Generally, the confinement potential proposed for such structure is a rectangular form. However, taking account of:

- The mixing of components of the core, well and shell in the interface and the presence of the impurities especially in growth period.

- The unphysical skiplike behavior of the potential energy and the effective mass in the interface region.

It is suitable for this particular problem, to adopt the following expression of the potential for the electron and the hole in which two confined parameters are taking account:

$$
V^{i}=\left\{\begin{array}{l}
V_{0}^{i}(x) r \leq R_{1} \\
a_{i} r^{2}+\frac{b_{i}}{r^{2}}-2 \sqrt{a_{i} b_{i}} R_{1} \leq r \leq R_{2} \quad i \equiv e, h \\
V_{0}^{i}(x) r \geq R_{2}
\end{array}\right.
$$

Taking account of $V^{i}\left(R_{1}\right)=V^{i}\left(R_{2}\right)=V_{0}^{i}(x)$ the parameters $a_{i}$ and $b_{i}$ are obtained as:

$$
\left\{\begin{aligned}
a_{i} & =\frac{V_{0}^{i}(x)}{\left(R_{2}-R_{1}\right)^{2}} \\
b_{i} & =\frac{R_{1}^{2} R_{2}^{2}}{\left(R_{2}-R_{1}\right)^{2}} V_{0}^{i}(x)
\end{aligned}\right.
$$

$V_{0}^{i}(x)$ denotes the conductor band offset of the conduction band $\left(V_{0}^{e}(x)\right)$ and the valence band $\left(V_{0}^{h}(x)\right)$ expressed as: 


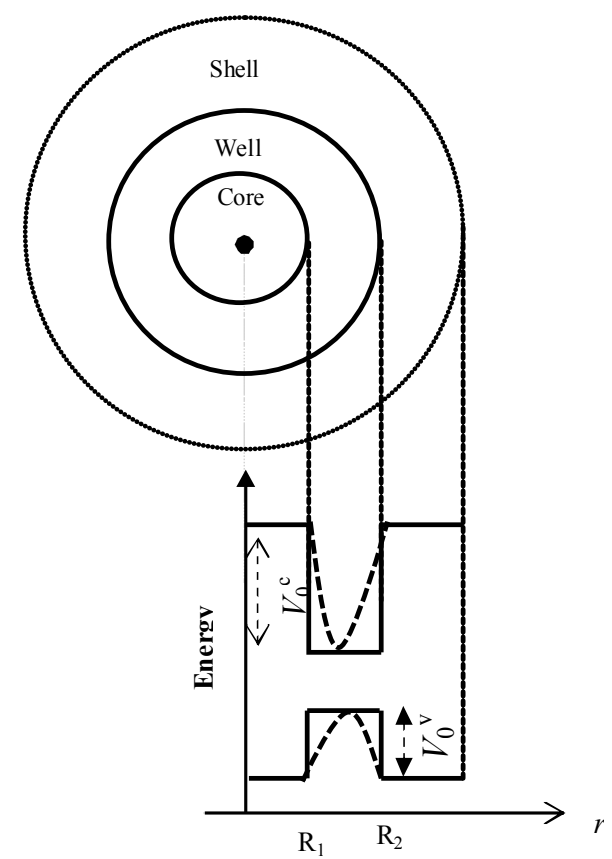

Fig. 1. The schematic description of the structure and the corresponding potential barrier confinement.

$$
\begin{gathered}
\left\{\begin{array}{l}
V_{0}^{e}(x)=0.7 \Delta E_{g}(x) \\
V_{0}^{h}(x)=0.3 \Delta E_{g}(x)
\end{array}\right. \\
\Delta E_{g}(x)=E_{g}^{G a N}-E_{g}^{I n_{x} G a_{1-x} N}(x)
\end{gathered}
$$

The band gap energy of the $\operatorname{In}_{x} \mathrm{Ga}_{1-x} \mathrm{~N}$ ternary alloy at room temperature is the subject of several experimental and theoretical studies. It's governed by the following formulas used in Ref. [16]:

$$
E_{g}^{(I n, G a) N}(x)=x \cdot E_{g}^{I n N}+(1-x) \cdot E_{g}^{G a N}-b \cdot x \cdot(1-x)(\mathrm{eV})
$$

$b=3.8$ is the bowing parameter taking account of the nonlinearity of the band gap as a function of the Indium fraction.

Within the framework of the effective-mass approximation and for a spherical symmetric potential, the separation of radial and angular coordinates leads to the wavefunction electron states confined in QDQW spherical nanoparticle as:

$$
\Psi_{n l m}(\vec{r})=R_{n l}(r) Y_{l m}(\theta, \varphi)
$$

$Y_{l m}(\theta, \varphi)$ is the spherical harmonic and $R_{n l}(r)$ is the radial wave-function. 


\section{II.2. Electron-hole transition in the well}

The effective-mass Hamiltonian of a single particle (electron, hole) in such structure in the absence of the hydrogenic impurity can be expressed as follows:

$$
H_{i}=-\frac{\hbar^{2}}{2} \nabla\left(\frac{1}{m_{i}^{*}}\right) \nabla+V^{i}
$$

$\nabla$ is the nabla operator which is used since the mass will be treated as a radial-dependent variable and $V^{i}$ is the potential barrier as mentioned above.

$$
m_{i}^{*}=\left\{\begin{array}{l}
m_{\mathrm{i}, \mathrm{GaN}}^{*} r \prec R_{1} \\
x \cdot m_{\mathrm{i}, \mathrm{InN}}^{*}+(1-x) \cdot m_{\mathrm{i}, \mathrm{GaN}}^{*} R_{1} \leq \mathrm{r} \leq R_{2} \\
m_{\mathrm{i}, \mathrm{GaN}}^{*} r \succ R_{2}
\end{array}\right.
$$

The stationary Schrodinger equation for the radial wave function has the form:

$$
\frac{d^{2} R_{i}(r)}{d r^{2}}+\frac{2}{r} \frac{d R_{i}(r)}{d r}+\left[\frac{2 m_{i}^{*}}{\hbar^{2}}\left(E^{i}-V^{i}(r)\right)-\frac{l(l+1)}{r^{2}}\right] R_{i}(r)=0
$$

To simplify the calculations, some parameters are introduced for both particles:

$$
\begin{gathered}
l(l+1)+\frac{2 m_{i}^{*} b_{i}}{\hbar^{2}}=2 s(2 s+1) \\
s=-\frac{1}{4}+\sqrt{\frac{m_{i}^{*} b_{i}}{2 \hbar^{2}}+\left(\frac{2 l+1}{4}\right)^{2}} \\
\sqrt{\frac{2 m_{i}^{*}}{a_{i} \hbar}}\left[E^{i}-2 \sqrt{a_{i} b_{i}}\right]=4(n+s)+3
\end{gathered}
$$

According to Ref [17] and taking into account the fact that the desired solution behavior for $r \rightarrow 0$ and $r \rightarrow \infty$ is given as follows:

$$
\left\{\begin{array}{l}
\exp \left(-\sqrt{\frac{m_{i}^{*} a_{i}}{2 \hbar^{2}}} r^{2}\right) r \gg a_{B} \\
\left(\frac{2 m_{i}^{*} a_{i}}{\hbar^{2}}\right)^{\frac{S}{2}} r^{2 S} r \ll a_{B}
\end{array}\right.
$$

Then, the radial wave function must be taken in the form:

$$
R_{i}(r)=\left(\frac{2 m_{i}^{*} a_{i}}{\hbar^{2}}\right)^{\frac{S}{2}} r^{2 S} U_{i}\left(-n, 2 S+\frac{3}{2}, \frac{\sqrt{2 m_{i}^{*} a_{i}}}{\hbar} r^{2}\right) \exp \left(-\sqrt{\frac{m_{i}^{*} a_{i}}{2 \hbar^{2}}} r^{2}\right)
$$

Note that $U_{i}(r)$ is the confluent hypergeometric function defined for a positive integer $n$.

An infinite set of equidistant values of energy levels of the particle (electron, hole) is obtained $(n=0,1,2 \ldots)$ and it is given as:

$$
E_{\mathrm{n}}^{i}=\sqrt{\frac{2 a_{i} \hbar^{2}}{m_{i}^{*}}}\left[2 n+1+\sqrt{\left(l+\frac{1}{2}\right)^{2}+\frac{2 b_{i} m_{i}^{*}}{\hbar^{2}}}\right]-\sqrt{a_{i} b_{i}}
$$


The sum of the confinement energies of the electron and hole and the bulk band-gap of the composite material gives the uncorrelated ground state electron-hole:

$$
E_{t r}=E_{0}^{e}+E_{0}^{h}+E_{g}(x)
$$

This bulk band-gap must be added since the confinement energies of the electron and the hole are obtained respectively relative to the conduction band and the valence band.

\section{RESULTS AND DISCUSSION}

To study the electron - hole transition, we have started to investigate the inner, the outer radii and the Indium composition effects on the electron and hole energies (not shown here). As we have mentioned above, in commonly used QDQW nanoparticles with narrow well layer, the $1 \mathrm{~S}$ state is the only confined state in the well. Fig. 2 shows the $1 S_{e}-1 S_{h}$ transition energy as a function of the ratio $\left(R_{2} / R_{1}\right)$ for Indium concentration equal to $25 \%$ and for three core radii.

It is shown that the transition energy decreases as a function of the radius ratio for all inner radii. For a fixed ratio, the transition energy decreases according to the inner radius, i.e., the quantization effect is more significant for smaller inner radius as expected.

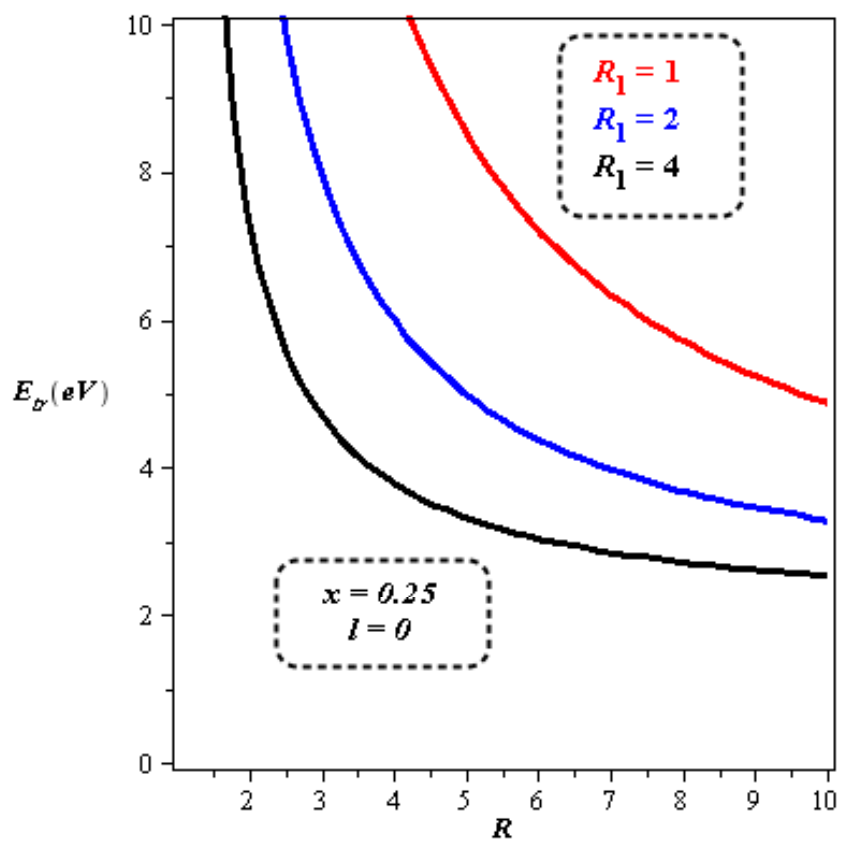

Fig. 2. The $1 S_{e}-1 S_{h}$ transition energy as a function of the outer radius and the inner radius ratio $\left(R=R_{2} / R_{1}\right)$ in $\mathrm{GaN}\left|\mathrm{In}_{0.25} \mathrm{Ga}_{0.75} \mathrm{~N}\right| \mathrm{GaN}$ spherical QDQW. The core radius effect is included. 
In Fig. 3, we present the barrier potential (Indium composition) effect on $1 S_{e}-1 S_{h}$ transition energy for two inner radii. The results show two different behaviors, i.e, a critical value of the ratio $\left(R_{c}\right)$ is obtained. For $R \leq R_{c}$, the transition energy increases as a function of the indium composition while it decreases for $R \geq R_{c}$. The results concerning the core radius equal to $R_{1}=2$ are also depicted in Fig. 3 (below). It is found that the critical ratio depends strongly of the inner radius. The more the core radius decreases, the more the critical radius increases. For the core radius equal to 3 and 2 the critical ratio is respectively 4.5 and 6.5 . This critical value of the ratio for which the behavior of the potential barrier (Indium composition) effect changes is known as a turning point.

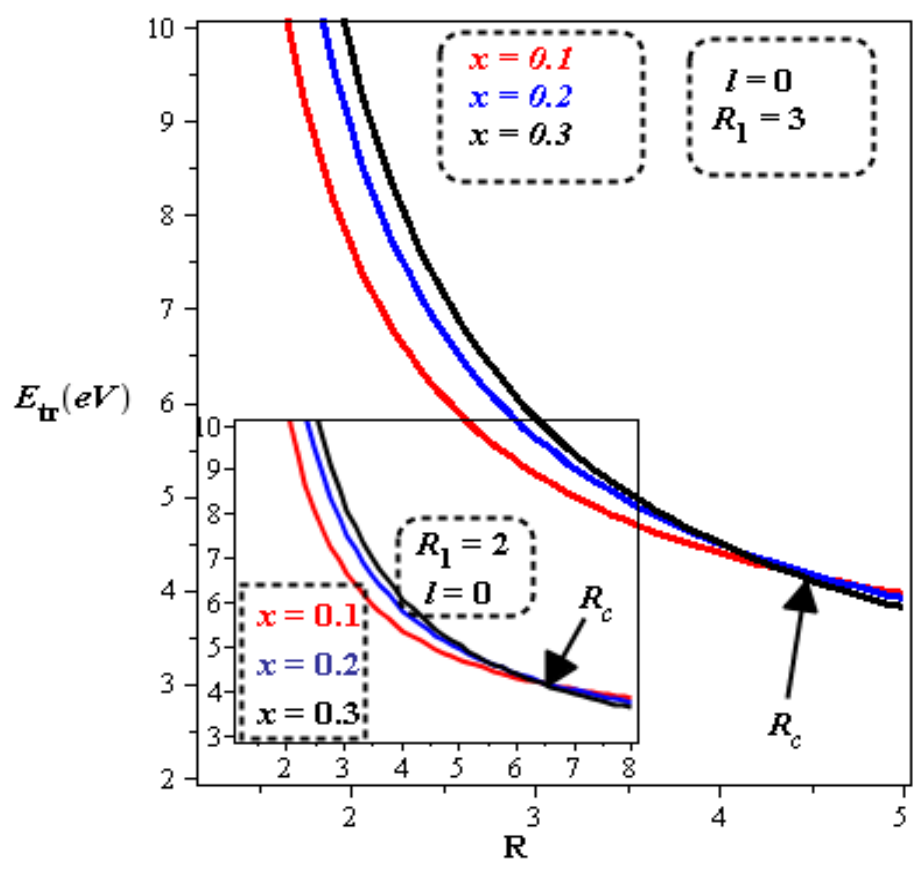

Fig. 3. The $1 S_{e}-1 S_{h}$ transition energy as a function of the radius ratio for two core radius in GaN|InGaN|GaN spherical QDQW. The potential barrier effect is included.

To the best of our knowledge, the turning behavior due to the barrier potential (Indium concentration) is not mentioned in all works cited above in the introduction. On the one hand, the effect of the potential barrier (composition) is not investigated in these works. On the other hand, this is due to the finite potential barrier and the wave function chosen in each work. We think also that the calculation method is very important. In the same sense, in our previous study concerning the potential barrier effect on an uncorrelated electron-hole recombination in (In, Ga)N/GaN strained quantum well (QW), this effect is not observed [18]. We have obtained that the indium composition effect is the same for electron-(heavy, light and crystal) holes transitions and any turning point is not obtained. 
Such turning behavior concerning the excited states energy and the binding energy under electric and magnetic fields as a function of the spherical quantum dot is reported by Sadeghi and Rezaie $[12,19]$. It has shown that the impurity energy changes from positive to negative when the dot radius is larger than $1.672 a^{*}\left(4.921 a^{*}\right)$ for $2 \mathrm{~S}(2 \mathrm{P})$ state. It is also obtained that for each dot radius; there is a critical electric (magnetic) field strength for which the excited state energies change from positive to negative. The turning point is obtained for electric field at $62.5 \mathrm{KV} . \mathrm{cm}^{-1}\left(1.52 \mathrm{KV} . \mathrm{cm}^{-1}\right)$ for $1.672 a^{*}\left(4.921 a^{*}\right)$ while is obtained for magnetic field at $\gamma_{0}=0.06$ for $1.653 a^{*}$ (2S state) and $\gamma_{0}=0.78$ for $4.921 a^{*}$ (2P state).

\section{CONCLUSION}

Uncorrelated $1 S_{e}-1 S_{h}$ electron-hole transition in Core|well|shell ( $\mathrm{GaN} \mid \operatorname{In}_{x} \mathrm{Ga}_{1-x}$ $\mathrm{N} \mid \mathrm{GaN}$ ) spherical QDQW nanoparticles is investigated within the framework of the effective mass scheme and the finite parabolic potential confinement barrier for which two confinement parameters are taking account. The inner, the outer radii and the Indium composition effects are reported. Two indium composition behaviors are obtained which are limited by the turning point.

\section{REFERENCES}

[1] A. Mews, A. Eychmuller, M. Giersig, D. Schooss and H. Weller, J. Phys. Chem. 98 (1994) 934-041.

[2] D. Schooss, A. Mews, A. Eychmuller and H. Weller, Phys. Rev. B49 (1994) 17072-17078.

[3] G. W. Bryant, Phys. Rev. B52 (1995) R16997-R17000.

[4] El Khamkhami, E. Feddi, E. Assaid, F. Dujardin, B. Stébé and J. Diouri, Phys. Low-Dim. Struct. 9 (2001) 131-142.

[5] A. Eychmuller, A. Mews and H. Weller, Chem. Phys. Lett. 208 (1993) 59.

[6] G. W. Bryant and W. Jaskolski, Phys. Stat. Sol. 224 (2001) 751-755.

[7] B. Little, M. El Sayed, G.W. Bryant and S. Burke, Chem. Phys. 114 (2001) 1813-1822.

[8] M. Millo, D. Katz, Y. W. Cao and U. Banin, Phys. Stat. Sol. 224 (2001) 271-276.

[9] F. Guffarth, R. Heitz, A. Schliwa, O. Stier, A.R. Kovsh, V. Ustinov, N.N. Ledentsov and D. Binbenrg, Phys. Stat. Sol. 224 (2001) 61-65.

[10] Y. Fang, M. Xiao and D. Yao, Physica E42 (2010) 2178-2183.

[11] E. M. Kazaryan, A. A. Kostanyan and R. G. Boghosyan, J. Phys. Conf. Series 350 (2012) 012020.

[12] E. Sadeghi and G. Rezaie, Pramana. J. Phys. 75 (2010) 749-755.

[13] H. El Ghazi, A. Jorio and I. Zorkani, Physica B (revised form 2013).

[14] J. W. Hauss, H. S. Zhou, I. Honma and H. Komiyama, Phys. Rev. B47 (1993) 1359.

[15] K. Chang and J. B. Xia, Phys. Rev. B57 (1998) 9780.

[16] H. El Ghazi, A. Jorio and I. Zorkani, Physica B410 (2013) 49-52

[17] L. D. Landau, E. M. Lifshitz, Quantum Mechanics: Non-Relativistic Theory, vol 3, $3^{\text {rd }}$ ed (1997) Pergamon Press

[18] H. El Ghazi, A. Jorio and I. Zorkani, Afr. Rev. Phys. 12 (2012) 237-241.

[19] E. Sadeghi, Physica E41 (2009) 1319-1322. 